\title{
La industria cerámica de la Comunidad Valenciana en el ámbito nacional y europeo
}

\author{
I. GIL, JJ GUARCH, C. ANDRÉS \\ Área de Organización de Empresas. Dpto. de Organización de Empresas, EF y C. E.T.S.I.I. de Valencia, 46022 Valencia
}

\begin{abstract}
La industria azulejera española englobada en uno de los sectores industriales más importantes y dinámicos del país, el cerámico, con una facturación global próxima al 1\% del PIB nacional, ha sido capaz de ocupar, (detrás de Italia) un papel de liderazgo internacional en la producción mundial de azulejos. No obstante las últimas cifras del sector, con mayores crecimientos de la cuota española $(14,39 \%)$ frente a la italiana $(3,84 \%)$ para 1997 respecto a la producción mundial, a la vez que los incrementos más importantes en cuota de mercado durante los cinco últimos años se han producido en la zona de Asia, protagonizados por China e Indonesia con un 6,2\% y 2,2\% respectivamente, junto con fuertes crecimientos de Brasil y México, hacen prever la aparición de cambios en la competitividad de los mercados que recomiendan una reflexión sobre los puntos fuertes y débiles de las empresas del sector, desde una perspectiva de recursos y capacidades, situadas fundamentalmente en la Comunidad Valenciana (Castellón), ya que su producción supone más del $80 \%$ del conjunto de la producción nacional.
\end{abstract}

Palabras clave: Producción cerámica, industrias azulejeras, industria, producción mundial, Comunidad Valenciana, Castellón

The “Comunidad Valenciana" ceramic tile industry in the Spanish and European market.

The Spanish Ceramic Tile Manufacturers has become one of the most dynamic and competitive business in Spain. One of the basic characteristics of the Spanish ceramic tile industry is its exporting vocation. Foreign trade is one of the pillars of the industry, which sets aside an each time greater percentage of its production for exportation. Approximately $50 \%$ of the World production is produced in Europe, with Italy and Spain producing 32.37\% in relation to the rest of the world, and $66.71 \%$ of the European production. Spain's share has been maintained at $14 \%$ of world production while Italy has lost some positions $(3,84 \%)$ for 1997. Outside Europe, Asia, Brazil, México and China are expanding. New Expectatives is the actual panorama of the International market. Around $80 \%$ of the workforce and $92 \%$ of production is concentrated in the Comunidad Valenciana, in particular in the province of Castellón (district of La Plana) will be necesary to study the strengths and weakness of these business.

Key Words: Ceramic production, Ceramic Tile Manufacturers, Industry, World production, Comunidad Valenciana, Castellón

\section{INTRODUCCION}

La producción del azulejo en general se encuentra concentrada en unos pocos países, fundamentalmente en Europa y Asia. Los cuatro primeros productores alcanzan aproximadamente la mitad de la producción mundial, los diez primeros suponen prácticamente el $70 \%$ y si miramos los veinte primeros países, su producción total alcanza cerca del $84 \%$, prácticamente la totalidad de las necesidades mundiales.

La cuota española respecto a la producción mundial alcanzó el 13,2\% durante el año 1996. Después de haber crecido durante los últimos años, a excepción de 1994, en 1996 se ha producido nuevamente una disminución del 0,3\% respecto a la producción mundial. La situación actual podría resumirse de la siguiente manera:

Italia, España y Brasil ocupan las tres primeras posiciones en producción y su situación se encuentra totalmente consolidada.

Mientras que España y Portugal han incrementado su cuota de mercado mundial un $0,9 \%$ y un $1,7 \%$ respectivamente el último año, el líder mundial (Italia) ha experimentado una fuerte pérdida de cuota de mercado del 6,1\%.
Los incrementos más importantes en cuota de mercado durante los últimos cinco años se han producido en la zona de Asia y han sido protagonizados por China e Indonesia con un $6,2 \%$ y $2,2 \%$ respectivamente.

España e Italia fabrican aproximadamente el 30\% de la producción mundial.

Los países de la UE producen una cifra cercana al 50\% del total mundial.

Brasil se muestra como un importante competidor tanto para España como para Italia; aunque la producción brasileña está destinada fundamentalmente al mercado interior siendo sus exportaciones pequeñas frente a los dos principales productores.

Algunos países con producciones mucho menores que las de los tres primeros están aumentando fuertemente su capacidad de fabricación: México, Taiwán, Tailandia e India, con un incremento en la variación de su producción durante los últimos cinco años superior al 100\%. 


\section{EL SECTOR EN LA UNIÓN EUROPEA}

La Unión Europea es el primer productor y consumidor de azulejos del mundo, es líder mundial en el sector del azulejo en virtud de la calidad de sus productos, así como por su producción, que representa una cuota de mercado mundial del 37,1\% con una producción en 1996 de 1.189,2 millones de metros cuadrados. La producción de la Unión Europea durante los últimos cinco años ha disminuido cerca de diez puntos; dicha pérdida se ha trasladado fundamentalmente en beneficio de la región asiática del planeta, que ha pasado de tener una cuota de mercado en 1991 del 21,3\% a tener una cuota del 30,3\% en 1996. Durante 1996 la UE tuvo un crecimiento muy pequeño en la producción del 2,2\%, casi cuatro veces menor que el crecimiento mundial. La explicación a la disminución se encuentra en la caída de la producción italiana junto con el acelerado crecimiento de China y los países del sudeste asiático.

La calidad del azulejo de la UE es muy apreciada, tal como explican los profesores Dalmau y De Miguel [1], debido básicamente a las grandes inversiones realizadas en investigación y desarrollo (I+D) durante las últimas dos décadas.

El consumo de azulejos depende en gran medida del sector de la construcción. La construcción sufrió una importante recesión a principios de la década de los ochenta y el consumo de revestimientos y pavimentos cerámicos pasó por un período de estancamiento. Durante la segunda mitad de la misma década el sector se recuperó apoyado por la mejora del sector de la construcción. El consumo durante la década de los noventa ha seguido una evolución positiva. El principal consumidor per capita es Italia; por el contrario, el Reino Unido es el país con menor consumo per cápita.

El número de empresas del sector del azulejo en la UE se encuentra en torno a las 650 y dan empleo a más de 70.000 trabajadores. La mayor parte de las empresas se encuentran en dos países: Italia, en la región de Sassuolo (provincia de Módena y Reggio Emilia) y en España, la mayor concentración de empresas se encuentra en la región de la "La Plana" de la Provincia de Castellón (Comunidad Valenciana).

La mayor parte de las exportaciones comunitarias van dirigidas a mercados que aprecian la calidad de los productos, por ejemplo los EE.UU, donde las exportaciones alcanzan cifras en torno al $20 \%$. Como se menciona en el libro " $E l$
Azulejo. Estudio sectorial" [1] la eliminación de las barreras arancelarias y no arancelarias existentes en muchos mercados proporciona una considerable expansión de las exportaciones de la industria comunitaria, aunque con distintos grados de penetración en función de las condiciones climáticas y del uso tradicional de los productos cerámicos en los diferentes mercados.

Italia y España, ocupan el primero y segundo puestos en producción mundial de azulejos respectivamente. Representan la mayor parte de la producción de la Unión Europea. La producción italiana en 1996 descendió un 1,37\%, aumentando en 1997 en un 3,84\%. Desde 1991 la producción italiana ha atravesado por años de bajo crecimiento, lo que le ha supuesto perder 6,1 puntos en su cuota mundial. En 1991 Italia representaba el $23,4 \%$ de la producción del mundo y en 1996 bajó hasta el 17,3\%.

España, a diferencia de Italia, ha incrementado su producción desde 1991 para ser en 1997 de un 14,39\% con una producción de 485 millones de $\mathrm{m}^{2}$. Francia, Alemania y Portugal se encuentran entre los quince principales países productores mundiales y sus producciones se encuentran en torno a la media de 58 millones de $\mathrm{m}^{2}$ en 1996, muy lejos de las producciones de Italia y España. Mientras que Francia y Portugal han experimentado una variación positiva en la producción durante los últimos cinco años, todo lo contrario ha ocurrido en Alemania, cuya producción ha descendido un 21,1\%.

\section{LOCALIZACIÓN DE LAS EMPRESAS EN LA COMU- NIDAD VALENCIANA}

La industria del azulejo en la Comunidad Valenciana se concentra fundamentalmente en la Provincia de Castellón tal como puede verse en la siguiente tabla comparativa del reparto geográfico de empresas entre 1994 y 1997:

Dicha zona, delimitada al Norte por las poblaciones de Alcora y Borriol, al Oeste por Onda, al Sur por Nules y al Este por la ciudad de Castellón de la Plana es justificada por De Miguel (1996)[2]:

La gran tradición empresarial en el sector.

La proximidad de los lugares de extracción de las arcillas.

La abundancia de empleados técnicos especializados, tanto en el ámbito de titulados como de personas con formación profesional en la especialidad azulejera que sólo existe en la

TABLA 1.

ProducCión POR ZONAS GEOGRÁfiCAs 1991-1996 (ProduCCión DE MILlONES DE M²)

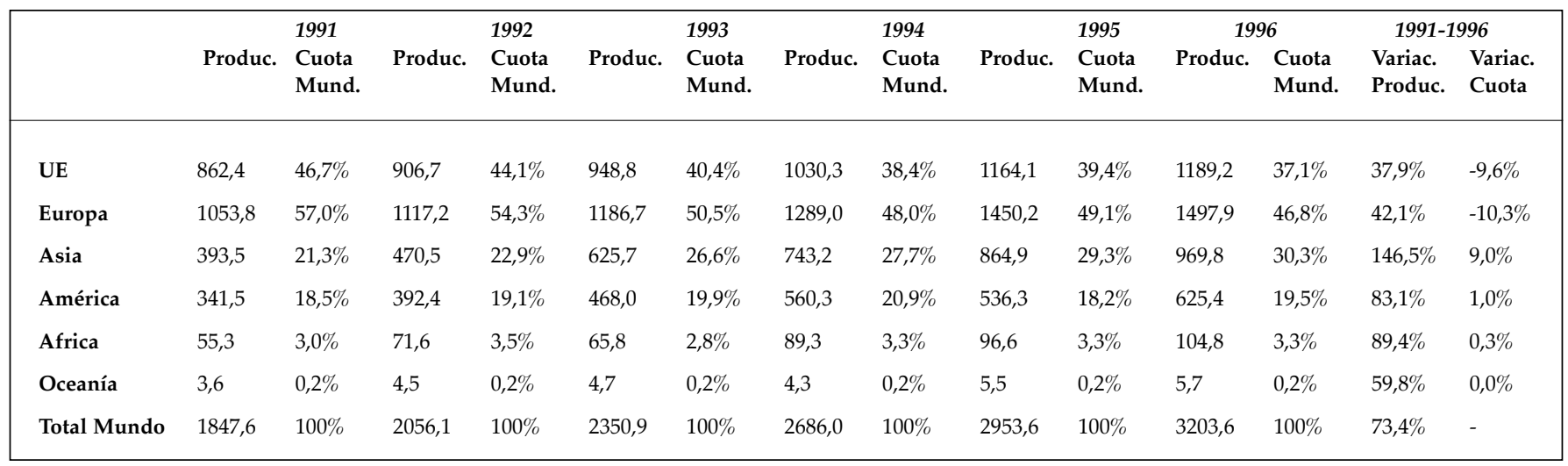


TABLA 2.

REPARTO GEOGRÁFICO DE LAS EMPRESAS (1994-1997)

\begin{tabular}{|llllll|}
\hline Provincia & 1994 & 1995 & 1996 & 1997 & $\begin{array}{l}\text { Crecimiento } \\
(1994-1997)\end{array}$ \\
\hline Castellón & $\mathbf{1 4 4}$ & $\mathbf{1 6 6}$ & $\mathbf{1 7 4}$ & $\mathbf{1 7 7}$ & $\mathbf{2 2 , 9 \%}$ \\
Barcelona & $\mathbf{1 2}$ & $\mathbf{1 2}$ & $\mathbf{1 2}$ & $\mathbf{1 2}$ & $\mathbf{0} \%$ \\
Valencia & $\mathbf{8}$ & $\mathbf{9}$ & $\mathbf{1 0}$ & $\mathbf{9}$ & $\mathbf{1 2 , 5} \%$ \\
Girona & $\mathbf{5}$ & $\mathbf{5}$ & $\mathbf{5}$ & $\mathbf{5}$ & $\mathbf{0} \%$ \\
Madrid & $\mathbf{2}$ & $\mathbf{2}$ & $\mathbf{2}$ & $\mathbf{2}$ & $\mathbf{0} \%$ \\
Teruel & $\mathbf{2}$ & $\mathbf{2}$ & $\mathbf{2}$ & $\mathbf{2}$ & $\mathbf{0} \%$ \\
Toledo & $\mathbf{1}$ & $\mathbf{1}$ & $\mathbf{1}$ & $\mathbf{2}$ & $\mathbf{1 0 0} \%$ \\
Alicante & $\mathbf{1}$ & $\mathbf{1}$ & $\mathbf{1}$ & $\mathbf{1}$ & $\mathbf{0} \%$ \\
Huelva & $\mathbf{1}$ & $\mathbf{1}$ & $\mathbf{1}$ & $\mathbf{1}$ & $\mathbf{0} \%$ \\
Granada & $\mathbf{1}$ & $\mathbf{1}$ & $\mathbf{1}$ & $\mathbf{1}$ & $\mathbf{0} \%$ \\
Jaén & $\mathbf{1}$ & $\mathbf{1}$ & $\mathbf{1}$ & $\mathbf{1}$ & $\mathbf{0} \%$ \\
Lugo & $\mathbf{1}$ & $\mathbf{1}$ & $\mathbf{1}$ & $\mathbf{1}$ & $\mathbf{0} \%$ \\
Pontevedra & $\mathbf{1}$ & $\mathbf{1}$ & $\mathbf{1}$ & $\mathbf{1}$ & $\mathbf{0} \%$ \\
Sevilla & $\mathbf{2}$ & $\mathbf{1}$ & $\mathbf{1}$ & $\mathbf{1}$ & $\mathbf{- 5 0 \%}$ \\
Tarragona & $\mathbf{1}$ & $\mathbf{1}$ & $\mathbf{1}$ & $\mathbf{1}$ & $\mathbf{0} \%$ \\
Valladolid & $\mathbf{1}$ & $\mathbf{1}$ & $\mathbf{1}$ & $\mathbf{1}$ & $\mathbf{0} \%$ \\
Zaragoza & $\mathbf{1}$ & $\mathbf{1}$ & $\mathbf{1}$ & $\mathbf{1}$ & $\mathbf{0} \%$ \\
Total & $\mathbf{1 8 5}$ & $\mathbf{2 0 7}$ & $\mathbf{2 1 6}$ & $\mathbf{2 1 9}$ & $\mathbf{1 8 , 4} \%$ \\
& & & & & \\
\hline
\end{tabular}

Fuente: Estudio sobre el sector, 1998 (ASCER)

provincia de Castellón.

Posibles economías de escala derivadas de una red empresarial (productores, distribuidores, talleres de mantenimiento, laboratorios de investigación aplicada, etc.).

El número de empresas en la Comunidad Valenciana y en particular en la Provincia de Castellón se ha ido incrementando gradualmente tal como se puede apreciar en los datos del sector aportados por ASCER (Tabla 3) [3], pasando el número de empresas de 144 en 1994 a 177 en 1997. A fecha de 1 de Septiembre de 1998 se contabilizaron 227 empresas en el sector, de las cuales 187 estaban asociadas a ASCER, lo que supone un $82,4 \%$ del total de empresas censadas y da una idea de la relevancia de la concienciación del colectivo como sector y el significativo valor del asociacionismo para estas empresas.

TABLA 3:

EVOLUCIÓN DEL NÚMERO DE EMPRESAS EN EL SECTOR

\begin{tabular}{|c|c|c|c|c|c|c|c|c|c|}
\hline \multirow[t]{2}{*}{ Año } & \multirow{2}{*}{$\begin{array}{r}1994 \\
\mathrm{~N}^{\circ} \\
\end{array}$} & \multicolumn{2}{|c|}{1995} & \multicolumn{2}{|c|}{1996} & \multicolumn{2}{|c|}{1997} & \multicolumn{2}{|c|}{$1998^{*}$} \\
\hline & & $\mathbf{N}^{\mathbf{o}}$ & $\Delta(\%)$ & $\mathbf{N}^{\mathbf{o}}$ & $\Delta(\%)$ & $\mathbf{N}^{\mathbf{o}}$ & $\Delta(\%)$ & $\mathbf{N}^{\circ}$ & $\Delta(\%)$ \\
\hline Empre. sector & 185 & 207 & 11,9 & 216 & 4,3 & 219 & 1,4 & 227 & 3,7 \\
\hline
\end{tabular}

Fuente: Estudio del sector, 1998 (ASCER) y elaboración propia.

*Nota: Datos referidos hasta el 1 de Septiembre de 1998.

\section{EMPLEADOS Y TAMAÑO DE LAS EMPRESAS}

Las empresas son generalmente pequeñas o medianas considerando el número de empleados como factor de medición.

Atendiendo a la información aportada por ASCER y considerando el número de empleados como factor de medición, se obtiene que, a nivel global las empresas del sector pertenecen a la categoría de Pymes ya que en 1996 más del 90\% no supe- raban los 250 trabajadores. En un porcentaje superior al 50\%, las empresas emplean a menos de 50 personas y son muy pocas las que emplean a más de 500 trabajadores. El mayor porcentaje es el formado por las empresas con una plantilla media de 25 a 50 empleados. Debe tenerse en cuenta que el número de trabajadores que emplea el sector de forma directa se eleva a 19.002, mientras que en el año 1989 esta cifra se encontraba en 15.000, aproximadamente.

TABLA 4.

NÚMERO DE EMPLEADOS POR EMPRESA (1994-1996)

\begin{tabular}{|lllll|}
\hline & \multicolumn{2}{c}{1994} & \multicolumn{2}{c|}{1996} \\
$\mathbf{N}^{\mathbf{0}}$ empleados & Empresas & Porcentaje & Empresas & Porcentaje \\
\hline Menos de 25 & 28 & $15,4 \%$ & 40 & $18,4 \%$ \\
$\mathbf{2 5 - 5 0}$ & 67 & $36,1 \%$ & 77 & $35,4 \%$ \\
$\mathbf{5 1 - 1 0 0}$ & 46 & $25,0 \%$ & 51 & $23,8 \%$ \\
$\mathbf{1 0 1 - 2 0 0}$ & 25 & $13,5 \%$ & 29 & $13,5 \%$ \\
$\mathbf{2 0 1 - 5 0 0}$ & 17 & $9,1 \%$ & 15 & $7,2 \%$ \\
Más de 500 & 2 & $1,0 \%$ & 4 & $1,8 \%$ \\
Total & 185 & $100 \%$ & 216 & $100 \%$ \\
\hline
\end{tabular}

Fuente: Estudio sobre el sector, 1998 (ASCER)

Las empresas Españolas del sector, a diferencia de las empresas italianas, sus principales competidoras, tienen un menor número de empleados.

\section{PRODUCCIÓN}

La producción de la Comunidad Valenciana supone más del $80 \%$ del conjunto de la producción nacional, manteniendo un crecimiento constante desde 1982.

TABLA 5.

EVOLUCIÓN DE LA PRODUCCIÓN ESPAÑOLA

\begin{tabular}{|c|c|c|c|}
\hline Años & $\begin{array}{l}\text { Producción } \\
\text { española } \\
\text { (millones de } m^{2} \text { ) }\end{array}$ & $\begin{array}{l}\text { Crecimiento } \\
\text { anual }\end{array}$ & $\begin{array}{l}\text { Cuota española } \\
\text { sobre la } \\
\text { producción } \\
\text { mundial }\end{array}$ \\
\hline 1977 & 98,3 & - & - \\
\hline 1978 & 97,8 & $-0,51 \%$ & - \\
\hline 1979 & 89,9 & $-8,08 \%$ & - \\
\hline 1980 & 116,0 & $29,03 \%$ & - \\
\hline 1981 & 112,5 & $-3,02 \%$ & - \\
\hline 1982 & 138,3 & $22,93 \%$ & - \\
\hline 1983 & 141,1 & $2,02 \%$ & - \\
\hline 1984 & 143,9 & $1,98 \%$ & - \\
\hline 1985 & 149,9 & $4,17 \%$ & - \\
\hline 1986 & 154,6 & $3,14 \%$ & - \\
\hline 1987 & 168,0 & $8,67 \%$ & - \\
\hline 1988 & 217,0 & $29,17 \%$ & - \\
\hline 1989 & 219,0 & $0,92 \%$ & - \\
\hline 1990 & 225,0 & $2,74 \%$ & $12,41 \%$ \\
\hline 1991 & 228,0 & $1,33 \%$ & $12,34 \%$ \\
\hline 1992 & 261,0 & $14,47 \%$ & $12,68 \%$ \\
\hline 1993 & 281,0 & $7,66 \%$ & $11,95 \%$ \\
\hline 1994 & 320,0 & $13,88 \%$ & $11,91 \%$ \\
\hline 1995 & 400,0 & $25,00 \%$ & $13,54 \%$ \\
\hline 1996 & 424,0 & $6,00 \%$ & $13,24 \%$ \\
\hline 1997 & 485,0 & $14,39 \%$ & - \\
\hline
\end{tabular}

Fuente: Estudio del sector, 1998 (ASCER) y elaboración propia. 
Los incrementos en la producción son más fuertes que en Italia y el resto del Mundo. Por significar una cifra, en 1997 la producción alcanzó los 485 millones de $\mathrm{m}^{2}$, con un crecimiento del $14,39 \%$ respecto a la producción del año anterior.

El tipo de producto fabricado a escala nacional se ha mantenido fiel en la mayoría de productos en el período 19941997, a excepción de las cuotas de producción de pavimentos y de prensados, que han aumentado en perjuicio de azulejos y extrudidos. A diferencia de España, en Italia se producen más revestimientos y menos gres porcelánico.

TABLA 6.

TiPOLOGÍA DE PRODUCTOS PRODUCIDOS (1994-1997)

\begin{tabular}{|c|c|c|c|c|}
\hline Tipos de productos & 1994 & 1995 & 1996 & 1997 \\
\hline Extruidos sin esmaltar & $3,46 \%$ & $3,64 \%$ & $3,23 \%$ & $3,70 \%$ \\
\hline Extruidos & $2,61 \%$ & $1,35 \%$ & $1,41 \%$ & $0,09 \%$ \\
\hline Gres porcelánico & $4,02 \%$ & $3,46 \%$ & $3,33 \%$ & $3,41 \%$ \\
\hline Azulejos & $49,59 \%$ & $49,14 \%$ & $47,09 \%$ & $46,21 \%$ \\
\hline Pavimentos esmaltados & $40,32 \%$ & $42,40 \%$ & $44,78 \%$ & $46,59 \%$ \\
\hline Total & $100 \%$ & $100 \%$ & $100 \%$ & $100 \%$ \\
\hline Prensados & $93,93 \%$ & $95,01 \%$ & $95,35 \%$ & $96,21 \%$ \\
\hline Extrudidos & $6,07 \%$ & $4,99 \%$ & $4,65 \%$ & $3,79 \%$ \\
\hline Total & $100 \%$ & $100 \%$ & $100 \%$ & $100 \%$ \\
\hline Esmaltados & $92,52 \%$ & $92,90 \%$ & $93,29 \%$ & $92,89 \%$ \\
\hline Sin esmaltar & $7,48 \%$ & $7,10 \%$ & $6,71 \%$ & $7,11 \%$ \\
\hline Total & $100 \%$ & $100 \%$ & $100 \%$ & $100 \%$ \\
\hline Azulejos & $49,59 \%$ & $49,14 \%$ & $47,09 \%$ & $46,21 \%$ \\
\hline Pavimentos & $50,41 \%$ & $50,86 \%$ & $52,91 \%$ & $53,79 \%$ \\
\hline Total & $100 \%$ & $100 \%$ & $100 \%$ & $100 \%$ \\
\hline Pasta roja & - & - & - & $83,81 \%$ \\
\hline Pasta blanca & - & - & - & $16,19 \%$ \\
\hline Total & - & - & - & $100 \%$ \\
\hline
\end{tabular}

Fuente: Estudio sobre el sector, 1998 (ASCER)

\section{PROVEEDORES Y MATERIAS PRIMAS}

El abastecimiento de materias primas básicas no constituye un problema para el sector, dado que las arcillas, caolines, feldespatos, etc., se hallan en la propia Comunidad Valenciana o en Provincias próximas como Teruel o Cuenca, por lo que no constituyen una partida importante en su estructura de costes.

Sin duda, tal como se comentó anteriormente, la proximidad de estas materias primas fue un elemento determinante para el nacimiento del sector en la Comunidad Valenciana y en concreto en la Provincia de Castellón.

A su vez subsectores fundamentales en el sector son el de esmaltes, fritas, barnices y colorantes, en los que es una tónica generalizada que participe un importante porcentaje de capital extranjero tratándose normalmente de empresas multinacionales.

Los esmaltes y colorantes son los materiales que constituyen el mayor porcentaje del coste total de la producción en ciertos tipos de fabricaciones. Ello es debido a que en su fabricación intervienen productos que hay que importar, caso del zircon, y que son difíciles de suministrar, soportando fuertes oscilaciones en los precios.

Respecto a la industria auxiliar de maquinaria, aunque se ha desarrollado cierta industria en nuestro país durante la década de los noventa, la dependencia respecto de la industria italiana es muy grande.

Y por último, en relación al suministro de la energía necesaria para la fabricación del producto. Las energías más utilizadas son el gas y la electricidad y dentro de esta última, en los últimos tiempos, la cogeneración.

\section{SISTEMAS DE DISTRIBUCIÓN}

Siguiendo nuevamente lo expuesto por Dalmau y De Miguel [1], se distinguen dos tipos de distribución en el sector:

Distribución interior, con los siguientes métodos:

a) Fabricante-Consumidor final

Se trata del método menos utilizado y se suele aplicar con los grandes clientes, con volúmenes de compra importantes.

TABLA 7.

Comparativa ItAliA-ESPAÑa EN PRODUCCIÓN DE REVESTIMIENTOS (R) Y PAVIMENTOS (P).

\begin{tabular}{|c|c|c|c|c|c|c|c|c|c|c|c|c|}
\hline & \multicolumn{6}{|c|}{1994} & \multirow{2}{*}{\multicolumn{2}{|c|}{$\begin{array}{l}1995 \\
\text { Italia }\end{array}$}} & \multicolumn{4}{|c|}{1996} \\
\hline & \multicolumn{2}{|c|}{ España } & \multicolumn{2}{|c|}{ Italia } & \multicolumn{2}{|c|}{ España } & & & \multicolumn{2}{|c|}{ España } & \multicolumn{2}{|c|}{ Italia } \\
\hline & Mill. $\mathrm{m}^{2}$ & $\%$ & Mill. $m^{2}$ & $\%$ & Mill. $\mathrm{m}^{2}$ & $\%$ & Mill. $m^{2}$ & $\%$ & Mill. $\mathrm{m}^{2}$ & $\%$ & Mill. $\mathrm{m}^{2}$ & $\%$ \\
\hline $\mathbf{R}$ & 159 & 49,6 & 138 & 27,2 & 197 & 49,1 & 155 & 27,6 & 200 & 47,1 & 145 & 26,2 \\
\hline $\mathbf{P}$ & 161 & 50,4 & 372 & 72,9 & 203 & 50,9 & 407 & 72,4 & 224 & 52,9 & 409 & 73,8 \\
\hline $\mathrm{TP}$ & 320 & 100 & 510 & 100 & 400 & 100 & 562 & 100 & 424 & 100 & 559 & 100 \\
\hline
\end{tabular}

Fuente: Estudio sobre el sector, 1998 (ASCER) (TP:Total Producción) 


\section{b) Fabricante-Gran almacenista-Consumidor final}

Los almacenes son aquellos locales en donde, además de revestimientos y pavimentos pueden comprarse sanitarios, grifería y otros elementos similares.

La razón de realizar este tipo de distribución es la conveniencia de ofrecer otros productos y no sólo los cerámicos, al objeto de satisfacer completamente las necesidades del cliente habitual.

La ventaja esencial del almacén es la proximidad al cliente. Si la fábrica de azulejos se encargara de realizar la venta directamente, en la mayoría de los casos existirían retrasos en la entrega del producto.

El material existente en el almacén es propiedad del almacenista; si fuese de la fábrica, el almacén acostumbra a llamarse depósito.

Existen empresas que buscan la integración con almacenistas, esto se realiza mediante una participación en el capital de la empresa almacenista por parte de la empresa fabricante.

En base a los almacenes observados en el sector los clasificaremos, sean grandes o pequeños, en:

\section{Exclusivistas}

Venden únicamente los productos de una sola empresa.

Distribuidores

Venden productos de distintas empresas, pudiendo o no disponer de existencias en el almacén.

\section{Mayoristas}

A diferencia de los distribuidores, los mayoristas siempre disponen de existencias en almacén.

c) Fabricante-Gran almacenista-Pequeño almacenista-

\section{Consumidor final}

Los pequeños almacenistas suelen atender pedidos del orden de hasta $100 \mathrm{~m}^{2}$ de producto cerámico (azulejo).

\section{d) Fabricante-Pequeño almacenista-Consumidor final}

Cuando el pequeño almacenista se dirige al fabricante es porque tiene en su cartera de clientes, compradores que le solicitan pedidos superiores a $100 \mathrm{~m}^{2}$.

En el último informe anual publicado por ASCER "El Azulejo. Estudio sectorial" (1998) [3] se señalan que los factores de elección de los fabricantes por los almacenistas son:

- Ser los fabricantes compatibles con los estilos de venta de los almacenistas.

- Dar buen servicio.

- Ofrecer un buen producto con el diseño apropiado.

- Disponer de una gama amplia de producto.

- No propiciar la competencia de distribuidores en una misma zona.

- Ayudar en la promoción.

- Seriedad.

- Fabricación de un producto con futuro.

\section{DISTRIBUCION EXTERIOR:}

La relevancia del mercado exterior como cliente va adquiriendo cada vez mayor importancia, alcanzando cifras del orden del $50 \%$ de la producción total actual.

La necesidad de buscar mercados extranjeros resulta evidente cuando el consumo interior resulta muy inferior a la producción alcanzada por el sector.

Durante la década de los noventa el azulejo exportado se ha caracterizado por la calidad y la variedad de formatos.

La comercialización en el exterior se realiza fundamentalmente a través de representantes, aunque existen otros métodos: almacenes de distribución propios, delegaciones exclusivas, sucursales y viajantes delegados de las empresas.

\section{EXPORTACIÓN}

Mientras que a finales de la década de los ochenta más de la tercera parte de la producción se destinaba a la exportación, esta cifra se ha ido incrementando y en la actualidad aproximadamente la mitad de la producción es destinada a la exportación, fundamentalmente como consecuencia de las mejoras en la calidad, el diseño y un fuerte aumento de la capacidad productiva.

Si se consideran solamente los resultados de 1997, las exportaciones se situaron en niveles del 52,82\% con aproximadamente 241,4 millones de metros cuadrados del conjunto total de ventas estimadas del sector, lo que supone un crecimiento de un $23,7 \%$ en $m_{-} ; \mathrm{y}$ la facturación en pesetas se incrementó en un $24,2 \%$, cifras que mejoraron significativamente los resultados de 1996.

La exportación ha pasado de vender azulejos en 1990 por valor de 65.400 millones de pesetas a ser en 1997 de 227.800 millones de ptas., lo que supone un espectacular crecimiento del $71,28 \%$. El precio medio de exportación ha seguido una evolución positiva, a excepción de la pequeña caída de 1995, el precio medio de exportación ha sufrido un incremento del 23,62\%, ello supone pasar de 721 ptas. $/ \mathrm{m}^{2}$ en 1990 a 944 ptas. $/ \mathrm{m}^{2}$ en 1997.

La mayor parte de las exportaciones se dirigen hacia países desarrollados, tal como justifican los profesores Dalmau y De Miguel [4] debido a las siguientes razones:

Son los que tienen capacidad para absorber las elevadas necesidades de exportación del sector azulejero español.

Valoran adecuadamente al aumento de la calidad y del diseño de los azulejos.

Son mercados marcados por la estabilidad, no así antiguos mercados del azulejo como Oriente Próximo y Medio o Sudamérica.

TABLA 8 .

EVOLUCIÓN DE LAS EXPORTACIONES EN EL PERÍODO 1990-1997

\begin{tabular}{|lllll|}
\hline Años Exportación & $\begin{array}{l}\text { \% exportación } \\
\left(\boldsymbol{m}^{2}\right) \times 10^{3}\end{array}$ & $\begin{array}{l}\text { Precio } \\
\text { sobre el total de } \\
\text { ventas estimadas }\end{array}$ & $\begin{array}{l}\text { Exportación } \\
\text { MPtas. }\end{array}$ \\
\hline $\mathbf{1 9 9 0}$ & 90721 & 40,32 & 721 & 65419 \\
$\mathbf{1 9 9 1}$ & 95103 & 43,6 & 769 & 73111 \\
$\mathbf{1 9 9 2}$ & 101548 & 41,75 & 849 & 86197 \\
$\mathbf{1 9 9 3}$ & 126499 & 43,97 & 881 & 111437 \\
1994 & 158443 & 46,80 & 904 & 143280 \\
$\mathbf{1 9 9 5}$ & 188117 & 48,93 & 899 & 169037 \\
1996 & 195207 & $50,94 \%$ & 939 & 183367 \\
1997 & 241397 & $52,82 \%$ & 944 & 2278081 \\
\hline
\end{tabular}

Fuente: Datos sobre el sector proporcionados por ASCER y elaboración propia. 
A continuación se muestra un análisis de las exportaciones españolas por países y áreas geográficas mundiales:

\section{UNIÓN EUROPEA}

Según la información sobre el sector aportada por ASCER, más de la mitad de las exportaciones, un $54,5 \%$ se dirigen a Europa fundamentalmente a cuatro países Reino Unido, Portugal, Francia y Alemania.

Los mercados naturales son Francia y Portugal, ya que por ejemplo, es más fácil llegar a Tolouse o Perpignan (Francia) que a Coruña, con una importante reducción de los costes de transporte.

\section{Reino Unido}

El Reino Unido es el principal mercado de la Unión Europea donde se dirigen las exportaciones. Las empresas nacionales son líderes en exportaciones a este país, aumentando en más de un $27 \%$ el valor de las ventas en 1997. El Reino Unido ha sido en 1997 el segundo destino de las exportaciones españolas en facturación mientras que ocupa el quinto lugar si la medición se realiza en metros cuadrados, a pesar de que el consumo de producto cerámico es relativamente bajo si lo comparamos con Alemania o Francia, una quinta parte y una tercera parte respectivamente. El precio medio de exportación es de los más elevados, en 1997 aumentó un 5,6\% por encima del crecimiento medio del año que se situó en un $0,5 \%$.

\section{Alemania}

Este país es el principal importador y el quinto consumidor mundial de baldosas cerámicas. Las exportaciones a este país sufrieron un fuerte retroceso en 1996 con una pérdida de mercado del $16 \%$ recuperándose modestamente en el último año hasta alcanzar valores de 13.016 metros cuadrados mientras que en 1994 se alcanzaron valores superiores a los 14.600 metros cuadrados. Como se puede observar en las tablas, Alemania ha perdido posiciones en la lista de los principales destinos de las exportaciones españolas. Si se considera que las importaciones alemanas desde España sólo supusieron en 1996 un 8,5\% del total, frente al 67,7\% de las italianas resulta evidente para las empresas del sector que tienen ante sí un mercado lleno de oportunidades.

A diferencia de otros mercados, los precios medios de exportación de España e Italia son muy similares. Resulta significativo que franceses y turcos mantengan cuotas de exportación muy cercanas a la mantenida por los españoles.

\section{Francia}

De igual manera que Alemania, Francia ha perdido posiciones en la clasificación de mercados de destino de las exportaciones españolas.

Se debe destacar que en 1997 se ha producido un incremento notable en las exportaciones a nuestro país vecino, con un aumento respecto a 1996 de más del 20\%. Francia pasa por ser el tercer país importador de producto cerámico del mundo. Mientras que Italia tuvo en 1997 una cuota de exportación del
$61,2 \%$, nuestro país se situó muy por debajo alcanzando cuotas del 15,3\%. El resto de países exportadores se situaron a mucha distancia de España, aproximadamente diez puntos por debajo. El precio medio de exportación de España era de 77,5\% del valor del precio medio italiano. El consumo per cápita francés era similar al alemán; ambos están por encima de los $2 \mathrm{~m}^{2} /$ persona y año, una de las mayores cifras del mundo sin considerar a España, Portugal e Italia, tradicionales consumidores y productores de baldosas cerámicas.

\section{Portugal}

Portugal es un país productor de baldosas cerámicas y sus importaciones se reducen aproximadamente a un $25 \%$ de lo que consume. Prácticamente todas las importaciones realizadas por Portugal provienen de España, baste mencionar que en 1996, el 97,3\% de las importadas tenía su origen en España. Durante los últimos dos años Portugal ha pasado a ser el segundo destino de las exportaciones por detrás de EE.UU. En lo referente al precio medio de exportación, este aumentó un 5,2\% en 1997.

\section{Otros países de la Unión Europea}

Especial mención debe realizarse respecto a Grecia, país con el que las exportaciones aumentaron en 1997 un 16,2\%, registro superior a la media de la UE que se situó en el último año en un $12,6 \%$. Grecia se caracteriza por ser un país importador; las importaciones suponen un $90 \%$ del consumo interno. El principal volumen proviene del país transalpino con un $62,1 \%$, mientras que España representa un porcentaje cercano al 33\%. En 1997, el precio medio de exportación a Grecia fue el más bajo de toda la UE y se situó en 680 ptas. $/ \mathrm{m}^{2}$. Respecto al precio medio de exportación italiano, el precio español supone el $68 \%$ del mismo. Otros países con compras significativas de producto cerámico español son: Los Países Bajos, Austria, Bélgica y Luxemburgo. Las cuotas de exportación de España en estos mercados son bajas, especialmente en el caso de Austria, con un escaso 3,8\% en 1996 frente al 79,3\% alcanzado por Italia.

\section{OTROS PAÍSES DE EUROPA}

No pueden olvidarse otros mercados que no pertenecen a la Unión Europea como son Polonia y Rusia que representan volúmenes importantes en la exportación española. La Europa Oriental representa el 10,7\% del destino de las exportaciones, cuota muy superior a la de principio de década que se situaba en un $0,6 \%$ de cuota total. Estos países han experimentado un notable incremento en el consumo de azulejos durante los últimos años. Polonia se situó como cuarto país importador del mundo en 1996 con 32,3 millones de $\mathrm{m}^{2}$, el $27,7 \%$ del producto importado tenía como origen España mientras que el 55,2\% provenían de Italia. Puede decirse que las exportaciones españolas a este país representan aproximadamente un $60 \%$ de las italianas. Con respecto al precio medio de exportación, resulta significativo que sea más caro en un $28 \%$ el español respecto al italiano.

Haciendo referencia a Rusia, España es el principal proveedor. Se puede decir que en 1991 este país ocupaba el puesto 96 en la lista de ventas exteriores españolas mientras que el últi- 
mo año se situó en la plaza 11; por tanto, el comportamiento ha sido espectacular. El precio medio de exportación se situó el último año en 1079 ptas. $/ \mathrm{m}^{2}$, bastante inferior al italiano 1647 ptas. $/ \mathrm{m}^{2}$.

Respecto al resto de países, las empresas españolas se caracterizan por tener una baja penetración. No son mercados muy grandes; en conjunto, las importaciones son superiores a los 30 millones de $\mathrm{m}^{2}$ e Italia es el principal exportador.

\section{PAÍSES DE ASIA}

España es el principal vendedor en esta región del planeta, destacando los países árabes e Israel. En conjunto las exportaciones crecieron un $12,6 \%$ en 1997; no obstante, es el menor incremento junto con las de la UE. La característica común a todos los mercados de esta región es la de los bajos precios medios de exportación.

\section{PAÍSES DE OCEANÍA}

ASCER lo califica como un continente que los empresarios deben hacer esfuerzos por explotar. Este mercado mejora considerablemente respecto a 1995 y 1996, años en que las ventas descendieron. Actualmente este mercado se reduce a Australia con una importación de más de 17 millones de $\mathrm{m}^{2}$. Los italianos son los principales valedores de este mercado aunque su precio medio de exportación sea un 30\% mayor. Países como Brasil, Indonesia, Tailandia y Turquía ocupan un $5 \%$ de las importaciones.

\section{PAÍSES DE AFRICA}

Los mercados africanos son pequeños y en conjunto, apenas suponen un $6 \%$ de las exportaciones totales españolas. Los principales países son Argelia y Sudáfrica.

\section{PAÍSES DE IBEROAMÉRICA}

Los países iberoamericanos han sido durante el último año el principal mercado de exportación por delante de los países del Este de Europa. En concreto, las exportaciones en $\mathrm{m}^{2}$ aumentaron un $99,3 \%$ en producto y un $69,4 \%$ en pesetas. El conjunto de las ventas a Iberoamérica fueron en 1997 de 16 millones de $\mathrm{m}^{2}$, una cuota del 6,6\% del total de exportaciones españolas, porcentaje similar al registrado por África. Sin embargo, mientras que las ventas al continente africano aumentaron un $14,4 \%$ en 1997, las exportaciones a Iberoamérica experimentaron un incremento de casi el 100\% en el mismo año. Los principales mercados son Colombia con 2,3 millones de $\mathrm{m}^{2}$ exportados, Chile y República Dominicana con 1,8 millones de $\mathrm{m}^{2}$, y Venezuela con 1,6 millones de $\mathrm{m}^{2}$.

México merece un tratamiento particular puesto que las exportaciones a éste país son muy elevadas aunque los últimos tres años han sufrido una importante disminución, mientras que en 1994 las ventas españolas fueron de 6,5 millones de $\mathrm{m}^{2}$, en 1995 bajaron a la cifra de $780.000 \mathrm{~m}^{2}$ y en 1997 se situaron en 3,5 millones de $\mathrm{m}^{2}$. El precio medio de exportación a México es el que más bajó en 1997, un 13,9\% inferior respecto a 1996.

\section{AMÉRICA DEL NORTE}

Como se indica en el último análisis realizado del sector por ASCER, Canadá es el único de los grandes países importadores del mundo en el que España no figura entre los tres primeros abastecedores de producto cerámico. En 1996 las exportaciones a este importante mercado estaban dominadas por Italia con un $43,1 \%$ de cuota, seguida de Turquía con un $13,6 \%$, Brasil con un 12,3\% y España con una cuota del 10,7\%.

EEUU es el principal mercado de exportación tanto español como italiano. En 1993, EEUU ya fue el principal destino de las exportaciones españolas, tanto en metros cuadrados como en pesetas, posición que ha alternado con Alemania y Arabia Saudí. El año 1997 fue el año más importante en ventas al mercado norteamericano, afianzándose en el primer puesto de la lista con un crecimiento en la facturación de las exportaciones de más del 34\%. El precio medio de exportación a este país crece a un ritmo ligeramente superior a la media mundial.

Italia domina este mercado norteamericano. Las exportaciones españolas son el $62 \%$ de las italianas, y la facturación menos de la mitad, un 40,2 \%. El precio medio de exportación italiano es un $54 \%$ mayor que el español. Un importante competidor del sector español es México que alcanzó una cuota del 23,5\% del mercado en 1996, casi seis puntos más que España que se situó en un 17,6\%. La proximidad geográfica hace que las empresas mexicanas se coloquen en una excelente posición competitiva. EEUU es el segundo país importador del mundo de baldosas cerámicas, por detrás de Alemania. Este dato, junto al importante crecimiento de su consumo e importación de productos cerámicos, el relativamente bajo consumo per cápita y el todavía importante margen de aumento de nuestra cuota de mercado frente a la de italianos y mexicanos convierten a EEUU como uno de los mercados más apetitosos para el sector español.

\section{PUNTOS FUERTES Y DÉBILES DEL SECTOR}

Los países competidores de España en el sector se caracterizan por tener en unos casos la mano de obra más cara, caso de Alemania, y en otros un coste en la mano de obra menor, como es el caso de Brasil o China.

La evolución del sector durante los últimos años ha conducido a un entramado de empresas de tamaño pequeño y medio, ágiles y dinámicas (PYMES), con instalaciones muy modernas en la mayoría de los casos.

La producción, en conjunto, debe seguir una tendencia positiva o creciente a medio y largo plazo si nos atenemos al mercado potencial: demanda de viviendas, obras públicas (metros, institutos, escuelas, pasajes, etc.), centros comerciales y otros lugares que precisan del azulejo en su construcción.

\section{Puntos fuertes:}

Materia prima disponible para los soportes, en cantidad y calidad.

Industria suministradora de esmaltes y barnices cercana, muy modernizada y con productos de alta calidad.

Personal excelentemente preparado, con experiencia de muchos años y profesionalidad.

Mercados consolidados, a lo largo y ancho de todo el mundo, gracias a una gestión sacrificada, creativa y bien llevada. 
Práctica imposibilidad de que vengan cantidades apreciables de productos de otros países, al existir en España una oferta de productos nacionales muy amplia, tanto en calidad como en precios.

Red de distribución consolidada y con buenas relaciones con los proveedores.

Centros de investigación muy bien preparados, tanto públicos como privados, en el sector de fritas y en el de azulejos.

Mano de obra más económica que la de algunos países con cuotas importantes en los mercados internacionales.

\section{Puntos débiles:}

Deficiente estructura empresarial, en ciertos casos, para poder competir con probabilidades de éxito en los mercados internacionales, más frecuentemente en las empresas con menor tamaño.

Predominio del sector de la construcción en la demanda: con ello la producción de azulejos queda sometida a las oscilaciones en la construcción de viviendas.

Importancia de los costes de transporte en el precio final al consumidor, debido al peso del $\mathrm{m}^{2}$ de producto.

Alto coste de colocación de pavimentos y azulejos.

Mano de obra más costosa que en otros países, sobre todo Extremo Oriente y Europa del Este; los cuales pueden competir frente a nuestros productos en determinados segmentos del mercado.

Falta, en algunos casos, de diseños originales y de calidad.

Cambios de la peseta en discordancia con la evolución de los costes. Este problema pensamos que quedará solucionado con la llegada de la esperada moneda única europea, el EURO.

Tratamiento fiscal inadecuado a la depreciación de la maquinaria, lo que perjudica la capitalización de las empresas a medida que pasa el tiempo.

El sector presenta atractivos para la inversión en empresas grandes, con capacidad exportadora y redes de distribución apropiadas, bien modernizadas y con productos de calidad. También para empresas de menor tamaño, pero concentradas en segmentos de mercado más reducidos en los que son muy competitivas. La atracción es menor para empresas de tamaño pequeño, con gama de productos amplia, en donde los costes de producción, a medio y largo plazo, les harán ser menos competitivas si no encuentran los segmentos de mercado apropiados. En este último caso, serían recomendables agrupaciones que hicieran más productivas las empresas participantes en la tarea común.

\section{Situación actual y tendencia}

Tal como se ha comentado en líneas anteriores, uno de los rasgos básicos de la industria española de baldosas cerámicas es su vocación exportadora. El comercio exterior es uno de los pilares de la industria, la cual destina cada vez un mayor porcentaje de su producción al exterior (un 52,8\% de las ventas totales en 1997). Estos datos demuestran la clara competitividad de las empresas españolas, que desde el primer momento han considerado los mercados exteriores como una pieza fundamental en su expansión y desarrollo.

La exportación española de baldosas, actualmente muestra una concentración en Europa. En Asia fundamentalmente en Países Arabes y Extremo Oriente (Hong Kong y Singapur).
Sin embargo la evolución del crecimiento futuro a medio plazo, de la exportación parece venir de la mano de Estados Unidos. Tal parece indicar la experiencia obtenida en la ciudad de Dallas con el proyecto "la casa del futuro", donde las revistas "Builders" y "Home" escogieron "Azulejos de España" para pavimentar los más de 4000 pies cuadrados de exposición.

Por otra parte España se sitúa a la cabeza de la tecnología mundial por el desarrollo continuo de programas de investigación, siendo las principales áreas de actuación: materias primas, proceso de fabricación y producto acabado. (Si bien hoy en día la maquinaria es mayoritariamente italiana, la tecnología más moderna es española).

En relación a el principal competidor, Italia, en 1997 se dieron señales de recuperación de su industria, (según comunicado de ASSOPIASTRELLE - Asociación de Fabricantes Italianos de Pavimentos y Revestimientos Cerámicos).

En el sector existe actualmente una tendencia continua a la realización de inversiones destinadas a reducir costes de energía, perfeccionar el proceso y el producto, aumentar el valor añadido y a disminuir o incluso a evitar el impacto ambiental.

Según un artículo publicado en la Revista "Cerámica Información" (Mayo 1998), cuando se consideran las causas principales de la crisis ecológica aparece el impacto de las actividades empresariales e industriales como factores fundamentales. La empresa tiene una responsabilidad con todos los grupos implicados en la actividad empresarial (o Stakeholders: trabajadores, distribuidores, proveedores y consumidores en general), y de hecho el fracaso de muchas empresas es debido a no tener en cuenta a estos grupos. Sólo dos de cada diez empresarios consultados (en el artículo) valoran positivamente el gasto en medio ambiente y ninguno considera que las mejoras medioambientales ayudan a mejorar las ventas o la imagen de su empresa.

En relación a datos de la producción en la industria se destacan 2 hechos: 1) la pérdida de peso del mercado germánico y 2) la confirmación de los Estados Unidos como principal país en consumo de cerámica española. La demanda de estos países, gira en torno a una acertada gestión medioambiental. Por eso, la industria cerámica, si quiere entrar en mayor profundidad en el mercado europeo y en el Norteamericano deberá plantearse un marketing verde, incorporando la "ecogestión".

En otro orden de cosas la tendencia al cambio tecnológico, como variable estratégica del sector, se orienta al progresivo incremento de gres porcelánico, buscando una mayor diferenciación y superiores niveles de calidad; la eliminación de la vía líquida para la incorporación de los esmaltes (deposición electroestática, prensado); la mejora de las técnicas decorativas así como la eliminación de problemas medioambientales, tal como se ha referido en el párrafo anterior.

El mercado obliga además a incorporar tecnologías de la información para soportar la gestión de los procesos, anteriormente citados, así como para potenciar su presencia en un mercado cada vez más interconectado, adaptando la oferta a las necesidades globales del sector, y obteniendo información clave que facilite su penetración en mercados tales como EE.UU, Canadá, ...

\section{LA CRISIS ASIÁTICA EN EL SECTOR}

La crisis del sureste asiático, que todavía sigue su curso, ha hecho revisar a la baja las expectativas de crecimiento de dis- 
tintos sectores industriales en todos los países del mundo, y el sector cerámico no es una excepción.

La inestabilidad financiera que comenzó en Tailandia en Junio de 1997, que ha hundido en la recesión a Asia y Rusia y ha frenado el crecimiento del resto de las zonas del mundo, tendrá un coste de entre 600.000 y 800.000 millones de dólares (Expansión, 1998). Este es el resultado de una desaceleración del PIB mundial, algo que gráficamente sería como si un país de las dimensiones económicas de Canadá dejase de producir durante "todo un año", (Fleming Larson, Co-responsable del servicio de Estudios del FMI).

La dependencia del exterior del sector cerámico obligará a :

Revisar a la baja las expectativas de crecimiento.

Buscar más activamente una penetración en el mercado norteamericano.

Los Países Bajos, Austria, Bélgica y Luxemburgo son un conjunto de países de la UE con compras significativas de producto cerámico, a la vez que muy sensibles a las repercusiones de las crisis dada su alta dependencia del exterior, no obstante las cuotas de exportación de España en estos mercados son bajas, especialmente en el caso de Austria (con un escaso 3,8\% en 1996 según ASCER), siendo las empresas azulejeras alemanas las que más han penetrado con éxito en estos países.

Por otro lado en las exportaciones a Rusia, calificadas en los últimos años como "espectaculares" (en 1991 ocupaba el puesto 96 en la lista de destino de las ventas exteriores españolas, mientras que en 1997 alcanzó la plaza 11, teniendo este último año un aumento de ventas del $67,7 \%$ ) provocará un cambio radical en esta tendencia cuyos efectos están aún por detallar.

Dado que España domina las ventas a los mercados asiáticos, en especial a países árabes e Israel, esta posición se verá afectada a la baja por la importante caída en las ventas del Este Asiático provocada por la crisis.

\section{CONCLUSION}

Las tendencias actuales en el sector se centran en medidas de ahorro basadas en:

La reducción del consumo de energía en las diferentes fases de producción (molienda, secado y cocción) y en general con la cogeneración anteriormente citada.

Al desarrollo de nuevos hornos; así el paso del túnel convencional al monoestrato de rodillos ha permitido reducir el consumo energético a través de una reducción en la temperatura de la cocción y en el tiempo de permanencia del horno, lo que adicionalmente prolonga la vida útil del material de enhornamiento.

La tecnología industrial aún habiendo alcanzado un alto nivel sigue siendo un factor potencial de ahorro. La deposi- ción vía seca de esmaltes la reducción de espesores son vias abiertas. Si que es importante incorporar tecnologías de información para controlar y potenciar los mercados tradicionales y acercarse a los potenciales. En estos aspectos hay una tendencia importante a la incorporación de sistemas integrados de gestión (ERP) que optimicen el uso de todos los recursos productivos y de gestión, y a utilizar herramientas de control de clientes y acercamiento a los mismos (comunicaciones e internet) posibilitando realizar negocios a cualquier hora, incluso cuando la empresa está cerrada.

\section{REFERENCIAS}

1. Dalmau, J.I., De Miguel, E., “El Azulejo. Estudio sectorial”. Ed. SPUPV, 1990

2. "Estructura de las PYMES en la Comunidad Valenciana". D. Enrique de Miguel Fernández. Editado por la Universidad Politécnica de Valencia, 1996

3. "Estudio del sector del azulejo", 1998. Ed. ASCER

4. Miquel, S., Dalmau, J.I. y De Miguel, E. “Análisis estratégico de los sectores industriales y del turismo en la comunidad Valenciana" Ed.SPUPV, 1993

5. “Anuario azulejo 1998”, CEVISAMA 98

Ed. Publica Sociedad Anónima, 1998

6. “Los Sectores de la Industria Valenciana (Apuntes). Juan I. Dalmau \& Joan J. Baixauli. Ed. Universidad Politécnica de Valencia, 1989

7. Informes de la Dirección General de Política de la PYME

"El euro y las PYMES"

"El euro y el comercio"

"Competitividad de las PYMES"

"Ventajas e inconvenientes para las PYMES"

"El problema informático del efecto 2000"

"Sistemas de ayuda a la PYMES de IPYME"

Editado electrónicamente por IPYME (Iniciativa de desarrollo empresarial). Dirección WWW: http:/ / www.ipyme.org

8. "La industria cerámica en España" M. Regueiro, E.Sánchez, V. Sanz, E.Criado, Bol. Soc. Esp. Cerám. Vidrio, 35(3) 439-352 (1996)

9. "Tendencias generales en la Industria Española de Cerámica y Refractarios", Bol. Soc. Esp. Ceram. Vidr. 33, 125-134 (1994),

10. “La PYME Industrial Española”. Ed. Price Waterhouse, 1995

11. "Cómo mejorar la gestión de las PYMES". Editado por Arthur Andersen y Expansión

12. Tirado, C., Granger, J.R., Nieto, M. ,“La PYME del siglo XXI”. Ed. FUNDESCO, 1995

13. “Los servicios avanzados de telecomunicaciones y su influencia en la industria”. Organismos FUNDESCO-MINER. Ed. Economía Industrial, 1995

14. Gil Pechuán, I., "Las Empresas del Sector Metal-Mecánico ante las T.I." Ed.IMPIVA, 1998

15. "Informe sobre la Industria Española. 1996. Sectores y Empresas Industriales". Ed. MINER. 1997

16. "Informe sobre la Industria Española. 1995. Sectores y Empresas Industriales". Ed. MINER. 1996

17. “Informes de la Organización de Cooperación y Desarrollo Económico. Las Nuevas Tecnologías de la Década de los Noventa”. Ed. OCDE, 1990

Recibido: 18-2-99

Aceptado: 12-3-99 